\title{
A Consensus Model to Detect and Manage Noncooperative Behaviors in Large-Scale Group Decision Making
}

\author{
Iván Palomares, Luis Martínez, Member, IEEE, and Francisco Herrera, Member, IEEE
}

\begin{abstract}
Consensus reaching processes in group decision making attempt to reach a mutual agreement among a group of decision makers before making a common decision. Different consensus models have been proposed by different authors in the literature to facilitate consensus reaching processes. Classical models focus on solving group decision making problems where few decision makers participate. However, nowadays, societal and technological trends that demand the management of larger scales of decision makers, such as e-democracy and social networks, add a new requirement to the solution of consensus-based group decision making problems. Dealing with such large groups implies the need for mechanisms to detect decision makers' noncooperative behaviors in consensus, which might bias the consensus reaching process. This paper presents a consensus model suitable to manage large scales of decision makers, which incorporates a fuzzy clustering-based scheme to detect and manage individual and subgroup noncooperative behaviors. The model is complemented with a visual analysis tool of the overall consensus reaching process based on self-organizing maps, which facilitates the monitoring of the process performance across the time. The consensus model presented is aimed to the solution of consensus processes involving large groups.
\end{abstract}

Index Terms-Consensus, e-democracy, fuzzy clustering, group decision making (GDM), preference relation, self-organizing maps (SOMs), social networks.

\section{INTRODUCTION}

D ECISION making processes are one of the most frequent mankind activities in daily life. The need for multiple views in decision making makes group decision making (GDM) increasingly necessary in many societies and organizations. GDM problems can be defined as decision situations where a group of decision makers or experts try to achieve a common solution to a problem consisting of two or more possible solutions or alternatives [1]. In real-world GDM problems, dif-

Manuscript received July 5, 2012; revised January 8, 2013; accepted April 19 , 2013. Date of publication May 16, 2013; date of current version May 29, 2014 This work was supported in part by the Research Project TIN-2009-08286 and European Regional Development Fund.

I. Palomares and L. Martínez are with the Department of Computer Science, University of Jaén, Jaén 23071, Spain (e-mail: ivanp@ujaen.es; luis.martinez@ujaen.es).

F. Herrera is with the Department of Computer Science and Artificial Intelligence, University of Granada, 18071 Granada, Spain, and also with the Faculty of Computing and Information Technology—North Jeddah, King Abdulaziz University, 21589 Jeddah, Saudi Arabia (e-mail: herrera@ decsai.ugr.es).

Color versions of one or more of the figures in this paper are available online at http://ieeexplore.ieee.org.

Digital Object Identifier 10.1109/TFUZZ.2013.2262769 ferent situations might usually occur, such as collaboration and competitiveness among individuals, compatible or incompatible proposals, etc. Some guiding rules, including the majority rule, minority rule, and unanimity [2], have been proposed to support decision making in such situations. For instance, the majority rule is classically the most usual rule to deal with GDM problems in democratic systems [3].

Traditionally, GDM problems have been solved by applying a selection process to choose the best alternative or subset of alternatives, paying no attention to the level of agreement achieved among experts [4]. However, many real-world problems that affect entire groups or societies (civil rights, raising taxes, political and religious issues, etc.) may require highly agreed decisions. Therefore, the need for making consensus-based decisions is becoming increasingly apparent in these contexts. Consensus reaching processes (CRPs) [2], [5] attempt to reach an experts' agreement before making a decision, thus yielding a more accepted solution by the whole group. In a CRP, experts discuss and modify their preferences, guided and supervised by a human figure known as moderator [6].

GDM and consensus models have been normally focused on dealing with a few number of decision makers [7]-[11], because classically in companies and administrations, important decisions have been made by one or a few number of them. However, current technological and societal demands have given birth to new paradigms in which decisions can be made taking into account a large number of decision makers (such as e-democracy [12], [13] and social networks [14]-[16]). Most current models are not appropriate to manage large groups, due to the high cost, complexity, and human supervision required. Additionally, a noticeable drawback usually found in such large groups is the presence of experts and subgroups of experts who present a behavior that does not contribute to achieve consensus [17], because they do not want to modify their initial position in order to achieve an agreement. In large groups, it is common that there exist several subgroups or coalitions of experts with similar interests. Some of these subgroups are prone to modify their preferences to achieve an agreement (they can be referred to as pro-coalitions), while some others do not modify their preferences or even do it on the contrary way to the remaining experts (they can be referred to as con-coalitions). Con-coalitions of experts introduce a bias in the collective opinion, since they move their preferences against consensus coordinately. Therefore, it would be advisable to detect and manage noncooperating individuals and subgroups [5], [17], with the aim of improving CRP performance. 
A visual analysis of the consensus evolution among decision makers' preferences throughout the discussion process, by means of a CRP monitoring tool to distinguish between those decision makers who move their preferences toward consensus and those ones who do not cooperate to achieve it, would also be very convenient to analyze consensus models. Self-organizing maps (SOMs) are a widely used tool capable of projecting highdimensional data (such as experts' preferences, for instance) into a low-dimensional space, maintaining the main topological properties of data to facilitate its visual analysis and interpretation [18]-[20].

In order to address the multiple challenges stated previously, in this paper, a consensus model capable of managing large groups of decision makers is proposed. Such a model incorporates an approach that classifies decision makers (based on their fuzzy preference relations) to detect noncooperative behaviors in CRPs and manage them. In order to achieve these objectives, fuzzy clustering techniques are used to facilitate the detection of noncooperating individuals or subgroups and deal with them accordingly. In line with the presented consensus model, we propose the use of a monitoring tool based on SOMs, which facilitates a visual analysis of experts' agreement evolution across the consensus process and their behavior.

This paper is organized as follows: In Section II, some preliminaries related to consensus processes in GDM, CRPs, fuzzy clustering techniques, and SOMs are reviewed. In Section III, the consensus model that deals with large scales of decision makers is presented, describing in detail the mechanisms to detect and manage experts' noncooperative behaviors. Section IV describes the use of SOM-based techniques to develop a monitoring tool to visualize the CRP performance. An illustrative example of the model's utility and applicability, including a visual analysis of the CRP, is shown in Section V. Finally, in Section VI, some concluding remarks are drawn.

\section{PRELIMINARIES}

In this section, we revise GDM problems, CRPs, and consensus models. We then briefly review fuzzy clustering techniques, which are the basis for the behavior detection scheme implemented in the proposed consensus model, and SOMs, which will be considered to propose a visual monitoring tool of the CRP performance.

\section{A. Group Decision Making}

GDM problems are characterized by the participation of two or more experts in a decision problem, where a set of alternatives or possible solutions to the problem are presented [1], [2]. Formally, the main elements found in any GDM problem are as follows.

1) A set $X=\left\{x_{1}, \ldots, x_{n}\right\},(n \geq 2)$ of alternatives to be chosen as possible solutions to the problem.

2) A set $E=\left\{e_{1}, \ldots, e_{m}\right\},(m \geq 2)$ of decision makers or experts, who express their judgements on the alternatives in $X$.

Each expert $e_{i}, i \in\{1, \ldots, m\}$, provides his/her opinions over alternatives in $X$ by means of a preference structure. One

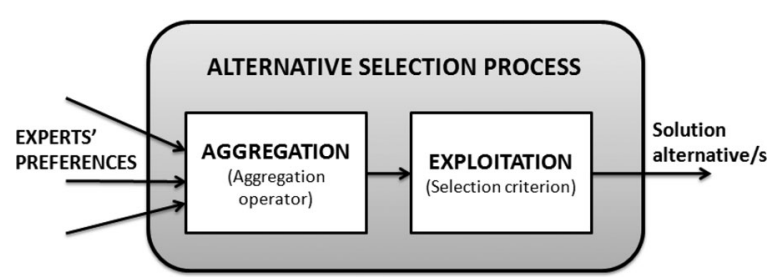

Fig. 1. Selection process in GDM problems.

of the most usual preference structures in GDM problems under uncertainty is the so-called preference relation [21], [22]. More specifically, fuzzy preference relations have proved to be especially effective to deal with uncertain information. They are defined as follows.

Definition 1 (see [23]): Given an expert $e_{i} \in E, i \in$ $\{1, \ldots, m\}$ and two different alternatives $x_{l}, x_{k} \in X ; l, k \in$ $\{1, \ldots, n\}(l \neq k)$, a fuzzy preference relation's assessment on the pair $\left(x_{l}, x_{k}\right)$, denoted as $p_{i}^{l k} \in[0,1]$, represents the degree of preference of alternative $x_{l}$ with respect to alternative $x_{k}$ assessed by expert $e_{i}$ so that $p_{i}^{l k}>1 / 2$ indicates that $x_{l}$ is preferred to $x_{k}, p_{i}^{l k}<1 / 2$ indicates that $x_{k}$ is preferred to $x_{l}$, and $p_{i}^{l k}=1 / 2$ indicates indifference between $x_{l}$ and $x_{k}$.

Definition 2 (see [21] and [24]): A fuzzy preference relation $P_{i}$ associated with expert $e_{i}, i \in\{1, \ldots, m\}$, on a set of alternatives $X$ is a fuzzy set on $X \times X$, which is characterized by the membership function $\mu_{P_{i}}: X \times X \longrightarrow[0,1]$. When the number of alternatives $n$ is finite, $P_{i}$ is represented by an $n \times n$ matrix of assessments $p_{i}^{l k}=\mu_{P_{i}}\left(x_{l}, x_{k}\right)$ as follows:

$$
P_{i}=\left(\begin{array}{ccc}
- & \ldots & p_{i}^{1 n} \\
\vdots & \ddots & \vdots \\
p_{i}^{n 1} & \ldots & -
\end{array}\right) .
$$

Assessments $p_{i}^{l l}, l \in\{1, \ldots, n\}$, situated in the diagonal of the matrix, are not defined, since an alternative $x_{l}$ is not assessed with respect to itself.

The solution to a GDM problem may be obtained either by a direct approach, where the solution is immediately obtained from experts' preferences, or by an indirect approach, where a social opinion is computed to determine the chosen alternative/s [4]. Regardless of the approach considered, it is necessary to apply a selection process to solve the GDM problem, which usually consists of two main phases (see Fig. 1) [25]: 1) an Aggregation phase, where experts' preferences are combined; and 2) an Exploitation phase, which consists of obtaining an alternative or subset of alternatives as the solution to the problem.

\section{B. Consensus Reaching Processes and Consensus Models}

The resolution of GDM problems by applying a selection process solely does not always guarantee that the decision would be accepted by all experts in the group, since some of them might consider that their opinions have not been sufficiently considered. In order to achieve a solution to the GDM problem which is accepted by the whole group, CRPs have been paid great attention as part of the decision process. Consensus can be 


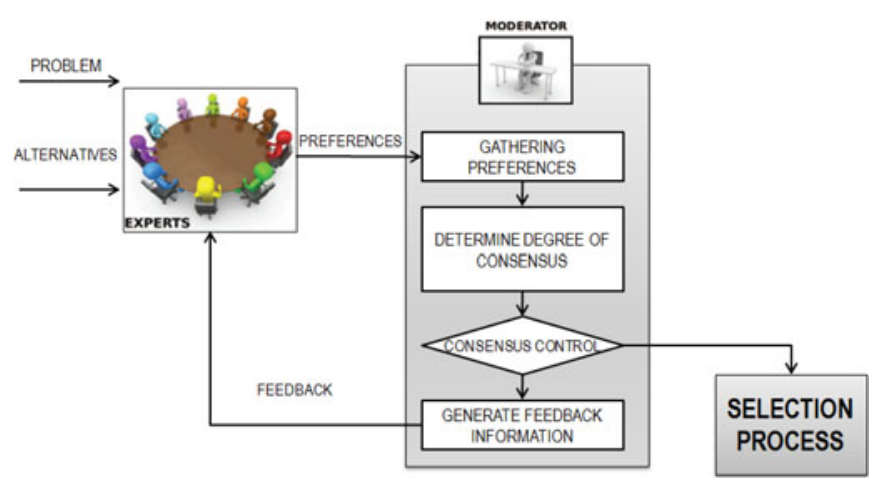

Fig. 2. General consensus process scheme in GDM problems.

understood as a state of mutual agreement among members of a group, in which the decision made satisfies all of them [2], [5]. Reaching a consensus usually requires that experts modify their initial opinions, making them closer to each other and toward a collective opinion which must be satisfactory for all of them.

The notion of consensus can be interpreted in different ways, ranging from consensus as total agreement to more flexible approaches [9], [26]. Consensus as a total agreement, where all experts achieve a mutual agreement in all their opinions, may be quite difficult to achieve in practice, and in cases that it could be achieved, the cost derived from the CRP would be unacceptable, and it might have been sometimes achieved under a normative point of view, through intimidation or other social strategies [17]. Subsequently, more flexible notions of consensus have been proposed to soften the strict view of consensus as a total agreement [26], [27], considering different degrees of partial agreement among experts to decide about the existence of consensus. One of the most widely accepted approaches for a flexible measurement of consensus is the so-called notion of soft consensus, which was proposed by Kacprzyk [1]. This approach introduces the concept of fuzzy linguistic majority, which establishes that consensus exists if most experts, participating in a problem, agree on the most important alternatives. Soft consensus-based approaches have been used in different GDM problems providing satisfactory results [28]-[30].

The process to reach a consensus in GDM problems is a dynamic and iterative discussion process [5], which is frequently coordinated by a human figure known as moderator, who is responsible for supervising and guiding experts in the overall process, as well as giving them advice to modify their opinions [6]. A general scheme of the phases required for conducting CRPs, which is depicted in Fig. 2, is briefly described below.

1) Gather preferences: Each expert provides moderator a preference structure with his/her opinion on the existing alternatives.

2) Determine degree of consensus: The moderator computes the level of agreement in the group by means of a consensus measure [26], usually based on different similarity measures and aggregation operators [31].

3) Consensus control: The consensus degree is compared with a threshold level of agreement desired by the group. If such degree is enough, the group moves on to the se- lection process; otherwise, more discussion rounds are required.

4) Generate feedback information: The moderator identifies furthest preferences from consensus and gives experts some pieces of advice, suggesting them how to modify their opinions and make them closer. Afterward, a new round of discussion begins with the gathering preferences phase.

In order to deal with CRPs, a large number of theoretical consensus models have been proposed in the literature by different authors [5], [8], [9], [11], [32]-[34]. These models have been designed to deal with GDM problems where small groups of decision makers participate, as has traditionally occurred in most companies and organizations, where decisions were delegated to one or, at the most, a low number of them.

However, new trends stemming from current demands in societal and technological contexts, such as e-democracy [12], [13] and social networks [14]-[16], make necessary to cope with consensus challenges in order that CRPs would be suitable to deal with larger scales of decision makers participating in the GDM problem [35], which implies a higher cost and complexity in such processes.

\section{Fuzzy Clustering}

Clustering is a widely used methodology, which is categorized as an unsupervised machine learning technique, aimed to data analysis and interpretation [36]. The problem of clustering consists in separating a set of data objects into a number of groups so-called clusters, based on a measure of similarity, so that data objects within the same cluster are more similar to each other than data objects belonging to different clusters [37]. Usually, each cluster is represented by a prototype or cluster center that characterizes all data objects belonging to such a cluster. Many clustering algorithms compute these cluster centers as the centroid of data belonging to the cluster considered.

Traditional or crisp clustering methods, such as k-means [38], are partitioning methods, i.e., each data object is assigned to one and only one cluster. Since this may not always provide a convincing representation of data, fuzzy clustering methods based on fuzzy set theory [39] have been later proposed under the assumption that data objects may belong to multiple clusters with different degrees of membership [37]. Fuzzy clustering methods are objective function-based methods which seek to find cluster centers for a predefined number $N$ of fuzzy clusters (for the sake of brevity, they will be referred to as clusters in the rest of this paper) and assign data objects a fuzzy membership degree to each cluster, during an iterative process aimed to minimize a predefined loss function [36], [40].

One of the most popular fuzzy clustering algorithms is the fuzzy c-means (FCM) algorithm [41], consisting in an optimization process where both cluster centers and data objects are iteratively updated until a locally optimal solution is found (which occurs when the variation between cluster membership degrees in two consecutive iterations of the algorithm approaches zero).

Algorithm 1 shows the basic steps in the standard FCM algorithm, defined according to our purpose of solving 


Algorithm 1 Fuzzy C-Means (FCM) Algorithm applied to
experts' fuzzy preference relations

1. Set the number of cluster centres $N,(N \geq 2)$, and degree of fuzziness $b$.

2. Initialize $N$ clusters $C_{h}, h \in\{1, \ldots, N\}$, by means of a cluster initialization technique.

3. while the stopping condition is not reached. do

4. Compute membership degrees of each preference relation $P_{i}$ to each cluster centre $C_{h}, \mu_{C_{h}}\left(P_{i}\right) \in[0,1]$, as follows:

$$
\mu_{C_{h}}\left(P_{i}\right)=\frac{\left(1 / d\left(P_{i}, C_{h}\right)\right)^{1 /(b-1)}}{\sum_{u=1}^{N}\left(1 / d\left(P_{i}, C_{u}\right)\right)^{1 /(b-1)}}
$$

5. Update cluster centres $C_{h}$ :

$$
C_{h}=\frac{\sum_{i=1}^{m} \mu_{C_{h}}\left(P_{i}\right) P_{i}}{\sum_{i=1}^{m} \mu_{C_{h}}\left(P_{i}\right)}
$$

6. end while.

GDM problems with fuzzy preference relations, assuming the following.

1) Considering the scope and purpose of this paper, the set of data objects is formed by all experts' preferences $P_{1}, \ldots, P_{m}$; therefore, $P_{i}$ is regarded as a data object. As a result, cluster centers $C_{h}, h=1, \ldots, N$ also consist of fuzzy preference relations.

2) Parameter $b,(b>1)$ indicates the degree of fuzziness of clusters. The larger $b$, the fuzzier the clusters [41]. A common value for this parameter is $b=2$.

3) A cluster initialization technique is required to set initial values for cluster centers $C_{h}$. Different cluster initialization techniques to perform this task can be found in the literature [42], [43].

4) Experts' fuzzy membership degrees to each cluster $\mu_{C_{h}}\left(P_{i}\right)$ are computed by using similarity measures, which are based on distance metrics. The distance between preference $P_{i}$ and cluster center $C_{h}$ is denoted as $d\left(P_{i}, C_{h}\right)$, and it will be introduced in Section III.

5) Further detail about the specific stopping condition considered in our proposal will be given in Section III-B.

\section{Self-Organizing Maps}

SOMs are a learning tool used in exploratory data mining, due to its prominent visualization properties [20], [44]. They were introduced by Kohonen [18] as a type of unsupervised learning algorithm based on neural networks [45], which is one of the most popular unsupervised learning methods for constructing topographic maps, i.e., low-dimensional (usually 2-D or 3-D) visualizations of high-dimensional data.

In the SOM algorithm, a set of $d$-dimensional training data is used to iteratively modify connections between artificial neurons (with the same dimension $d$ ) situated in a rectangular- or hexagonal-shaped grid, which is progressively adapted to such data. For each data object in the training set, the most similar neuron to such data object, the so-called Best Matching Unit (BMU), must be found among all the artificial neurons in the grid. Connection weights in the BMU and its nearest neighboring neurons are updated upon the given data object. This process is iteratively conducted to progressively learn the structure of the whole SOM [36], [45]. The resulting SOM can then be used as a visualization surface to represent future sets of data objects in a low-dimensional space, preserving its main topological properties [18], [45].

Once constructed, the SOM can visualize high-dimensional datasets. There are multiple methods based on SOMs to visualize data, such as distance matrices, similarity coloring, data histograms, and PCA projections [19], the latter of which will be considered in this paper. Most of these methods can be used either for a 2-D or a 3-D visualization of data [20].

SOMs have proved themselves to be a useful tool in different data mining applications to obtain qualitative information, such as full-text and financial data analysis, cluster analysis, vector quantization and projection, etc., [19], [44].

\section{Consensus Model to Detect And Manage NONCOOPERATIVE BEHAVIORS}

In this section, a consensus model that is suitable to deal with a large number of decision makers in the resolution of GDM problems is presented. The main novelty of such a model is the approach to classify decision makers according to their preferences and detect individual and subgroup noncooperative behaviors in the CRP, based on fuzzy clustering techniques, as well as dealing with those experts who present such behaviors, with the aim of improving the overall CRP performance.

The consensus model description will be divided into three parts.

1) a general scheme of the model, according to the main phases conducted in CRPs (see Section III-A);

2) a fuzzy clustering-based method to classify experts' preferences and detect noncooperative behaviors (see Section III-B);

3) a scheme based on weights to manage noncooperating experts and subgroups of experts (see Section III-C).

Fig. 3 shows a scheme of the consensus model, whose main phases and modules are developed in the following sections.

\section{A. Consensus Model Scheme}

The proposed consensus model aims to serve as a guide to carry out the main tasks required to conduct CRPs, as stated in Section II-B. Such a model (see Fig. 3) extends the basic ideas of the ones previously proposed in [7] and [10] and incorporates additional modules to achieve our goal of detecting experts' noncooperative behaviors and dealing with them.

The consensus model design allows an easy automation of the human moderator tasks, thus removing his/her inherent subjective bias and facilitating the resolution of GDM problems with large groups of experts computationally. Let us remark that, regarding the scheme presented later to deal with noncooperating experts, which will be based on experts' importance weights, we propose that each expert $e_{i} \in E$ has an associated importance weight $w_{i} \in[0,1]$, which is initially $w_{i}=1, \forall i \in\{1, \ldots, m\}$, and may vary when the CRP goes on. Further detail on the meaning and use of such weights will be given in Section III-C. 


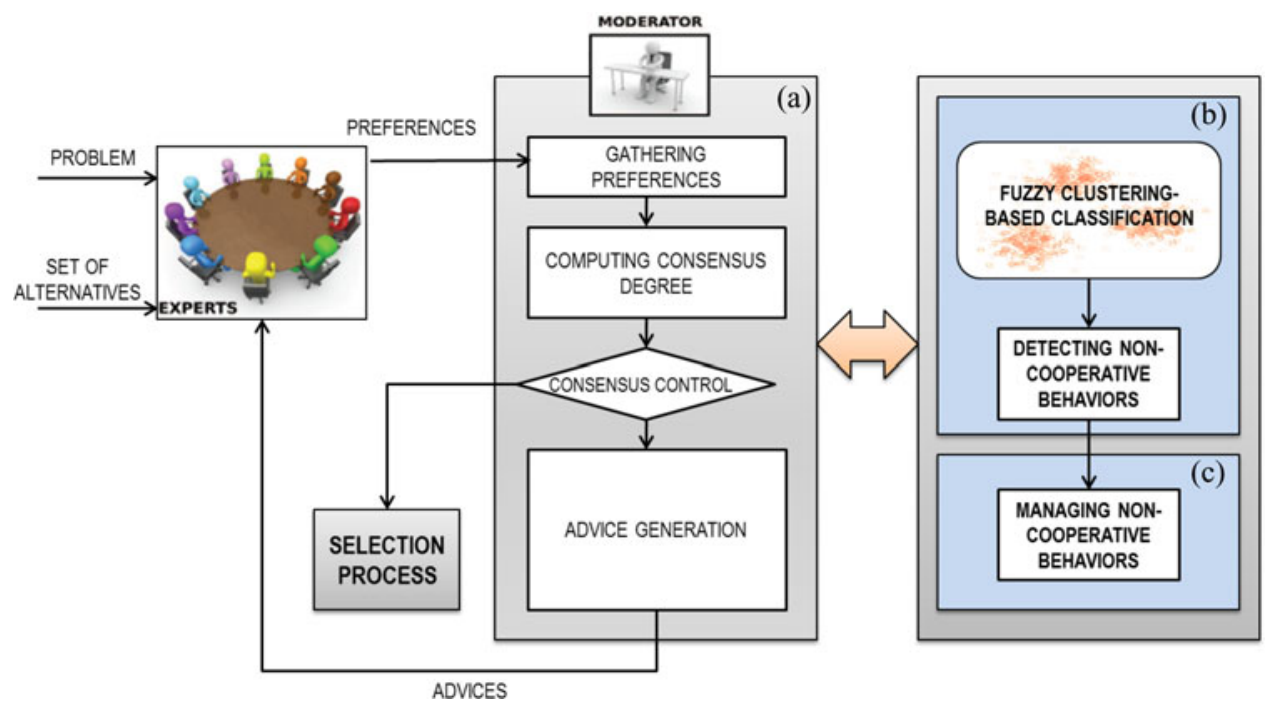

Fig. 3. Consensus model scheme.

In the following, the four basic phases of the proposed consensus model are described in detail.

1) Gathering Preferences: Each expert $e_{i} \in E$ provides his/her preference on alternatives in $X$ to the moderator, by means of a fuzzy preference relation $P_{i}=\left(p_{i}^{l k}\right)^{n \times n}$, consisting of a matrix of assessments $p_{i}^{l k}$ on each pair of alternatives $\left(x_{l}, x_{k}\right), l, k \in\{1, \ldots, n\}$. Consistency in preferences can be improved if experts provide reciprocal assessments, i.e., if $p_{i}^{l k}=p, p \in[0,1], l \neq k$, then $p_{i}^{k l}=1-p$.

2) Computing Consensus Degree: The moderator computes the level of agreement between experts by means of the following steps:

a) For each pair of experts $e_{i}, e_{j},(i<j)$, a similarity matrix $S M_{i j}=\left(s m_{i j}^{l k}\right)^{n \times n}$ defined by

$$
S M_{i j}=\left(\begin{array}{ccc}
- & \ldots & s m_{i j}^{1 n} \\
\vdots & \ddots & \vdots \\
s m_{i j}^{n 1} & \ldots & -
\end{array}\right)
$$

is computed. $s m_{i j}^{l k} \in[0,1]$ is the similarity degree between experts $e_{i}$ and $e_{j}$ in their assessments $p_{i}^{l k}, p_{j}^{l k}$, which is obtained by means of a similarity function as follows [8]:

$$
s m_{i j}^{l k}=1-\left|\left(p_{i}^{l k}-p_{j}^{l k}\right)\right|
$$

b) A consensus matrix $C M=\left(\mathrm{cm}^{l k}\right)^{n \times n}$ is computed by aggregating similarity matrices, taking into account the importance weights $w_{i j} \in[0,1]$ associated with each pair of experts $\left(e_{i}, e_{j}\right), i<j$. Each element $\mathrm{cm}^{l k} \in[0,1], l \neq k$, is computed as the weighted average of similarity degrees:

$$
c m^{l k}=\frac{\sum_{i=1}^{m-1} \sum_{j=i+1}^{m} w_{i j} s m_{i j}^{l k}}{\sum_{i=1}^{m-1} \sum_{j=i+1}^{m} w_{i j}} .
$$

Further detail about weights $w_{i j}$ and the way they are computed upon single experts' weights $w_{i}, w_{j}$ can be found in the scheme to manage noncooperating experts described in Section III-C. Notice here that if all experts are given equal importance weights, $\mathrm{cm}^{l k}$ can be computed as

$$
c m^{l k}=\frac{\sum_{i=1}^{m-1} \sum_{j=i+1}^{m} s m_{i j}^{l k}}{m(m-1) / 2}
$$

with $m(m-1) / 2$ being the number of different pairs of experts $\left(e_{i}, e_{j}\right)$ in the group [in both (4) and (5)].

c) Consensus degree is computed at three different levels [8], [10]:

i) Level of pairs of alternatives $\left(c p^{l k}\right)$ : obtained from $C M$ as $c p^{l k}=c m^{l k}, l, k \in$ $\{1, \ldots, n\}, l \neq k$.

ii) Level of alternatives $\left(c a^{l}\right)$ : The level of agreement on each alternative $x_{l} \in X$ is computed as

$$
c a^{l}=\frac{\sum_{k=1, k \neq l}^{n} c p^{l k}}{n-1}
$$

iii) Level of preference relation (overall consensus degree, $c r)$ :

$$
c r=\frac{\sum_{l=1}^{n} c a^{l}}{n}
$$

3) Consensus Control: The overall consensus degree $\mathrm{cr}$ is compared with a consensus threshold $\mu \in[0,1]$ established $a$ priori. If $\mathrm{cr} \geq \mu$, then the CRP ends and the group moves on to the selection process; otherwise, more discussion rounds are required. A parameter Maxround can be used to limit the number of discussion rounds conducted in the cases that consensus cannot be achieved.

4) Advice Generation: If $c r<\mu$, the moderator advises experts to modify their preferences in order to increase the level of agreement in the following rounds. Since this is 
the last phase of each discussion round in the CRP, the schemes to detect and manage noncooperative behaviors must be applied in a parallel way (see Sections III-B and C) so that experts' importance weights $w_{i}$ will be updated before initiating the following round of discussion. Three steps are considered in the advice generation phase.

a) Compute a collective preference and proximity matrices for experts: A collective preference $P_{c}$ is computed for each pair of alternatives by aggregating experts' preference relations:

$$
p_{c}^{l k}=\frac{\sum_{i=1}^{m} w_{i} p_{i}^{l k}}{\sum_{i=1}^{m} w_{i}}
$$

where $w_{i} \in[0,1]$ is the importance weight assigned to $e_{i}$ (see Section III-C). If all experts have the same importance, then $p_{c}^{l k}$ can be computed as

$$
p_{c}^{l k}=\frac{\sum_{i=1}^{m} p_{i}^{l k}}{m} .
$$

Once computed $P_{c}$, we have all the necessary data to initiate the fuzzy clustering-based algorithm to classify and group experts according with their preferences, as it will be shown in Section III-B.

b) A proximity matrix $P P_{i}=\left(p p_{i}^{l k}\right)^{n \times n}$ between each expert's preference relation and $P_{c}$, which is defined by

$$
P P_{i}=\left(\begin{array}{ccc}
- & \ldots & p p_{i}^{1 n} \\
\vdots & \ddots & \vdots \\
p p_{i}^{n 1} & \ldots & -
\end{array}\right)
$$

is computed. Proximity values $p p_{i}^{l k}$ are obtained for each pair $\left(x_{l}, x_{k}\right)$ as follows:

$$
p p_{i}^{l k}=1-\left|\left(p_{i}^{l k}-p_{c}^{l k}\right)\right| .
$$

Proximity values are used to identify the furthest preferences from the collective opinion, which should be modified by some experts.

c) Identify preferences to be changed $(C C)$ : Pairs of alternatives $\left(x_{l}, x_{k}\right)$ whose consensus degrees $c a^{l}$ and $c p^{l k}$ are not enough are identified:

$$
C C=\left\{\left(x_{l}, x_{k}\right) \mid c a^{l}<c r \wedge c p^{l k}<c r\right\} .
$$

Afterward, the model identifies experts who should change their opinion on each of these pairs, i.e., those experts $e_{i}$ whose preference $p_{i}^{l k}$ on the pair $\left(x_{l}, x_{k}\right) \in C C$ is furthest to $p_{c}^{l k}$. An average proximity $\overline{p p}^{l k}$ is calculated to identify them as follows:

$$
\overline{p p}^{l k}=\frac{\sum_{i=1}^{m} p p_{i}^{l k}}{m} .
$$

As a result, experts $e_{i}$ whose $p p_{i}^{l k}<\overline{p p}^{l k}$ are advised to modify their assessment on pair $\left(x_{l}, x_{k}\right)$.

d) Establish change directions: Several direction rules are applied to suggest the direction of changes proposed to experts, in order to increase the level of agreement in the following rounds [10]. Here, an acceptability threshold $\varepsilon \geq 0$ which may take a positive value close to zero is introduced to allow a margin of acceptability when $p_{i}^{l k}$ and $p_{c}^{l k}$ are close enough to each other.

i) DIR.1: If $\left(p_{i}^{l k}-p_{c}^{l k}\right)<-\varepsilon$, then expert $e_{i}$ should increase his/her assessment on the pair of alternatives $\left(x_{l}, x_{k}\right)$.

ii) DIR.2: If $\left(p_{i}^{l k}-p_{c}^{l k}\right)>\varepsilon$, then expert $e_{i}$ should decrease his/her assessment on the pair of alternatives $\left(x_{l}, x_{k}\right)$.

iii) DIR.3: If $-\varepsilon \leq\left(p_{i}^{l k}-p_{c}^{l k}\right) \leq \varepsilon$, then expert $e_{i}$ does not need to modify his/her assessment on the pair of alternatives $\left(x_{l}, x_{k}\right)$.

\section{B. Noncooperative Behavior Detection}

Once described the main phases of the proposed consensus model, here we define a method to identify those experts and subgroups of them who do not tend to modify their initial preferences to achieve a consensus or might move such preferences against it, either individually or coordinately. We aim to develop such a method by applying the FCM algorithm for fuzzy clustering [41], in order to classify experts based on their fuzzy preference relations $P_{i}$. Once applied the FCM algorithm, the definition of several rules is proposed, based on cluster similarity, cluster distance metrics, and fuzzy logic. These rules must be checked before deciding about the existence of the aforementioned behaviors.

The detection scheme is conducted once for each round in the discussion process, after the collective preference $P_{c}$ for that round is obtained during the Advice Generation phase of the basic consensus model scheme (see Section III-A). Let $t \in$ $\{1, \ldots$, Maxround -1$\}$ be the current discussion round of the CRP. From now onwards, experts' preference values in round $t$ will be denoted as $P_{i}^{t}, i=1, \ldots, m$, and cluster centers in such a round will be denoted as $C_{h}^{t}, h=1, \ldots, N$.

The description of the proposed detection method is organized into three parts:

1) application and settings of the FCM algorithm to classify experts;

2) rules for the detection of subgroup behaviors contrary to consensus achievement (con-coalitions);

3) rules for the detection of individual behaviors contrary to consensus achievement (considered as outliers).

1) FCM Algorithm Settings: First, the FCM algorithm is applied on experts' preferences in the current CRP round $t$. Several specifications and variations respect to FCM will be considered here, and they are described as follows:

1) FCM parameters: Without loss of generality, a fuzziness degree $b \approx 2$ is usually taken.

2) Cluster initialization: As reviewed in Section II-C, the first phase in the FCM algorithm consists in initializing clusters, i.e., assigning each of them a cluster center $C_{h}^{t}$ based on an initialization technique. We consider the method proposed by Katsavounidis et al. in [43] to define the initialization scheme described below for $N$ clusters $(N>$ 2): 


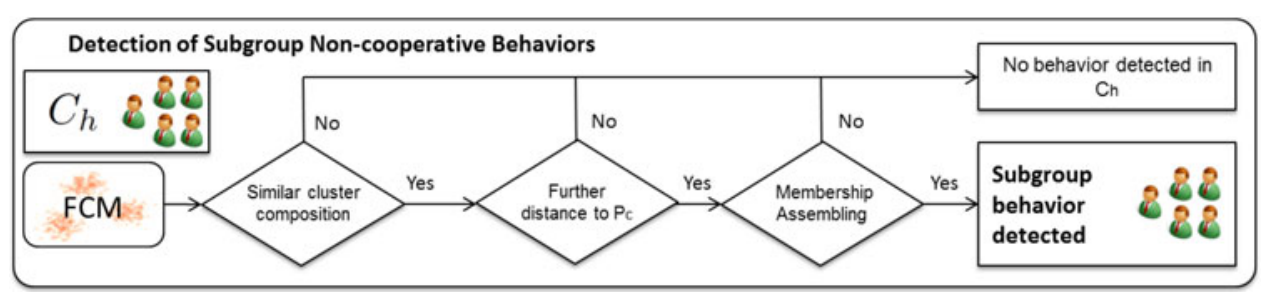

Fig. 4. Scheme of the method to detect subgroup noncooperative behaviors.

a) The first cluster is initialized by assigning the collective preference in the current round, $P_{c}^{t}$, to cluster center $C_{1}^{t}$.

b) Initialize the second cluster center $C_{2}^{t}$ as the expert preference $P_{i}^{t}$ which is farthest from $P_{c}^{t}$.

c) For $C_{h}^{t}(h \geq 3)$, compute the minimum distance between each of the remaining experts' preferences $P_{i}^{t}$ and all current initial cluster centers, and find the expert preference whose minimum distance is the largest one, i.e., the one which accomplishes $\max _{i}\left(\min _{u<h} d\left(P_{i}^{t}, C_{u}^{t}\right)\right)$. Assign it to $C_{h}^{t}$.

d) Repeat the third step until all $N$ clusters are initialized.

3) Update process: Cluster centers $C_{h}^{t}(h \geq 2)$ and cluster membership degrees $\mu_{C_{h}^{t}}\left(P_{i}^{t}\right)$ are updated iteratively, as shown in Algorithm 1. Notice here that $C_{1}^{t}$ is not updated in order to preserve $P_{c}^{t}$ as the center of one of the clusters once applied the FCM algorithm, since it will play an essential role in the subsequent detection scheme.

4) Distance metrics: In order to compute distances between preference relations (both experts' preferences and cluster centers indistinctly), the following normalized Minkowski-based distance measure [36] is considered:

$$
d\left(P_{i}^{t}, C_{h}^{t}\right)=p \sqrt{\sum_{l k, l \neq k}\left(p_{i}^{l k, t}-c_{h}^{l k, t}\right)^{p}}
$$

where $p>0$ and $l, k \in\{1, \ldots, n\}$.

5) Stopping criterion: The stopping condition considered is to finalize the update process when all clusters stabilize. This occurs when the variation in membership degrees between two consecutive iterations approaches zero. Formally, the iterative update process is stopped when

$$
\frac{\sum_{i=1}^{m} \sum_{h=1}^{N}\left|\mu_{C_{h}^{t}}^{y}\left(P_{i}^{t}\right)-\mu_{C_{h}^{t}}^{y-1}\left(P_{i}^{t}\right)\right|}{m \cdot N} \leq \epsilon
$$

where $y \in \mathbb{N}$ denotes the current iteration of the FCM algorithm, and $\epsilon$ is a threshold value (which should be close to zero) used as a stopping condition. As an optimization algorithm where a locally optimal solution is always found, FCM guarantees the necessary convergence to achieve this condition.

2) Detection of Subgroup Noncooperative Behaviors (ConCoalitions): Once executed the FCM algorithm, we proceed to apply a method to detect individual and subgroup noncooperative behaviors, which is aimed to facilitate the subsequent treatment of such experts, thus improving the performance of the CRP. Since a different rule-based scheme will be considered for each type of behavior (subgroup or individual), they will be explained separately. In this section, we present the scheme corresponding to the detection of subgroup noncooperative behaviors which, as stated in Section I, can be regarded in this paper as con-coalitions. This detection scheme is first applied in the second round of the CRP, because it requires comparisons between clusters obtained in the previous and current rounds of discussion, i.e., $t-1$ and $t$, and it is based on a set of three rules which must be checked for each cluster center $C_{h}^{t}, h \geq 2$, to decide about the existence of a subgroup behavior on it (see Fig. 4).

1) There exists a cluster with "similar" composition to $C_{h}^{t}$ in round $t-1$.

2) The distance between $C_{h}^{t}$ and $P_{c}^{t}$ increases.

3) Membership of experts to $C_{h}^{t}$ increases or membership to $P_{c}^{t}$ decreases.

The accomplishment of all these rules by a cluster $C_{h}^{t}$ can be assumed as a subgroup noncooperative behavior performed by a con-coalition of experts belonging to it, whose preferences must be given some treatment, as will be explained in Section III-C.

In the following, the rules are described in detail.

R1. Similar Cluster Composition: This rule is checked to determine whether a cluster is compound by the same experts across the time or not. To do this, the similarity between a given cluster $C_{h}^{t}(h \geq 2)$ determined in the current CRP round $t$, i.e., $t \in\{2, \ldots$, Maxrounds -1$\}$, and each cluster $C_{u}^{t-1}(u \geq 2)$ determined in the previous round, $t-1$, is computed. Two clusters $C_{h}^{t}$ and $C_{u}^{t-1}$ are considered to represent the same subgroup of experts, if experts' membership degrees to both of them, i.e., $\mu_{C_{h}}^{t}\left(P_{i}^{t}\right)$ and $\mu_{C_{u}}^{t-1}\left(P_{i}^{t-1}\right)$, have close values to each other, for all $e_{i} \in E$.

In order to decide whether cluster similarity is enough to assume analogous cluster composition, a similarity threshold $\kappa \in[0,1]$ can be defined. A cluster similarity measure $\operatorname{sim}\left(C_{h}^{t}, C_{u}^{t-1}\right)$ is proposed as follows:

$$
\operatorname{sim}\left(C_{h}^{t}, C_{u}^{t-1}\right)=1-\frac{\sum_{i=1}^{m} \Delta_{h u}^{t}\left(P_{i}\right)}{m}
$$

where $\Delta_{h u}^{t}\left(P_{i}\right) \in[0,1]$ is the variation in $P_{i}$ membership to both clusters, which is computed as

$$
\Delta_{h u}^{t}\left(P_{i}\right)=\left|\mu_{C_{h}}^{t}\left(P_{i}^{t}\right)-\mu_{C_{u}}^{t-1}\left(P_{i}^{t-1}\right)\right| .
$$

For a given cluster $C_{h}^{t}$, if $\exists C_{u}^{t-1}: \operatorname{sim}\left(C_{h}^{t}, C_{u}^{t-1}\right) \geq \kappa$, then $C_{h}^{t}$ and $C_{u}^{t-1}$ are assumed to represent the same cluster across time, due to their similar composition.

Remark 1: Since $\operatorname{sim}\left(C_{h}^{t}, C_{u}^{t-1}\right)$ takes values in the unit interval, the value fixed for similarity threshold $\kappa$ should be 


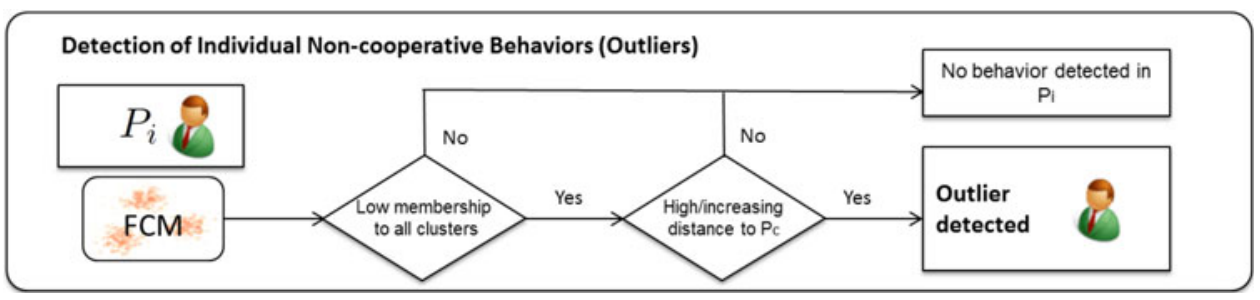

Fig. 5. Scheme of the method to detect individual noncooperative behaviors.

close enough to 1 in order to guarantee an effective detection of similar clusters in consecutive rounds of the CRP.

R2. Further Distance to $P_{c}$ : Based on the previous rule and assuming that $C_{h}^{t}$ and $C_{u}^{t-1}$ are similar enough to be considered the same cluster, distances between a cluster center and the collective preference (i.e., $C_{1}^{t}$ ) in rounds $t$ and $t-1$, which are denoted as $d\left(C_{1}^{t}, C_{h}^{t}\right)$ and $d\left(C_{1}^{t-1}, C_{u}^{t-1}\right)$, respectively, are computed by means of the distance measure shown in (13).

Let $\nu \in[0,1]$ be a parameter indicating a minimum distance between clusters, which should take a value close to zero so that a distance value lower than $\nu$ means that cluster centers are close enough to each other and no further detection process is required. If $d\left(C_{1}^{t}, C_{h}^{t}\right)>\nu$ and $d\left(C_{1}^{t}, C_{h}^{t}\right) \geq d\left(C_{1}^{t-1}, C_{u}^{t-1}\right)$, i.e., cluster centers $C_{1}^{t}$ and $C_{h}^{t}$ are not close enough to each other and distance between them increases as the CRP progresses, then some experts in cluster $C_{h}$ are presumably presenting a noncooperative behavior.

R3. Membership Assembling: This rule is checked to decide whether one of the following conditions occurs: 1) A subgroup of experts become more assembled around a cluster $C_{h}^{t}$ (i.e., their membership to the cluster increases), or 2) there is a lower concentration of experts around the collective opinion $P_{c}^{t}$. Assuming again that $C_{h}^{t}$ and $C_{u}^{t-1}$ are considered to be same cluster, let $S_{h}^{t}=\sum_{i=1}^{m} \mu_{C_{h}^{t}}\left(P_{i}^{t}\right)$ and $S_{u}^{t-1}=\sum_{i=1}^{m} \mu_{C_{u}^{t-1}}\left(P_{i}^{t-1}\right)$ be the sums of experts' membership degrees to cluster $C_{h},(h \geq 2)$, in rounds $t$ and $t-1$, respectively. Analogously, let $S_{1}^{t}=\sum_{i=1}^{m} \mu_{C_{1}^{t}}\left(P_{i}^{t}\right)$ and $S_{1}^{t-1}=\sum_{i=1}^{m} \mu_{C_{1}^{t-1}}\left(P_{i}^{t-1}\right)$ be the sums of experts' membership degrees to the collective preference in the aforementioned rounds.

If $S_{h}^{t}>S_{u}^{t-1}$, then experts are becoming more assembled around $C_{h}^{t}$. On the other hand, if $S_{1}^{t}<S_{1}^{t-1}$, then experts become less assembled around $C_{1}^{t} \equiv P_{c}^{t}$.

3) Detection of Individual Noncooperative Behaviors (Outliers): Here, the scheme corresponding to the detection of individual behaviors is described. Such behaviors must also be managed later to optimize the performance of the consensus process, and they are determined by preference relations that present a low membership to all clusters in the group; therefore, they can be viewed as outliers in the set of experts' preferences. This scheme is only applied toward the end of the CRP, i.e., when discussion between experts has already been developed and the consensus degree $\mathrm{cr}$ approaches the consensus threshold $\mu$. An additional consensus threshold $\gamma<\mu, \gamma \in[0,1]$ can be used to decide when the outlier detection mechanism is activated.
The following rules are checked to determine the existence of an individual noncooperative behavior associated with a preference relation $P_{i}^{t}$ (see Fig. 5):

R1. $P_{i}$ does not present a high membership to any cluster: A cluster membership threshold $\delta \in[0,1]$ is established. $P_{i}^{t}$ does not present a high membership to any cluster if $f$ $\mu_{C_{h}}^{t}\left(P_{i}^{t}\right)<\delta, \forall h \in\{1, \ldots, N\}$.

R2. High/increasing distance to $P_{c}$ : Distance to the collective preference increases or it is higher than the average distance between all experts' preferences and the collective preference, i.e., either one of the following conditions holds:

a) $d\left(P_{i}^{t}, C_{1}^{t}\right)>\bar{d}$.

The average distance to the collective preference, which is denoted by $\bar{d}$, is computed as follows:

$$
\bar{d}=\frac{\sum_{i=1}^{m} d\left(P_{i}^{t}, C_{1}^{t}\right)}{m}
$$

b) $d\left(P_{i}^{t}, C_{1}^{t}\right)>d\left(P_{i}^{t-1}, C_{1}^{t-1}\right)$.

Remark 2: The rules described previously have been proposed to detect the specific type of subgroup and individual noncooperative behaviors this paper focuses on. However, the proposed model offers enough flexibility to introduce new rules and/or extend the current ones, if any new kind of behavior would be considered.

\section{Managing Noncooperative Behaviors}

Once individual and subgroup behavior detection mechanisms have been presented, it is necessary to define how to manage experts involved in such behaviors. There exist different proposals in the literature concerning this issue, for instance, discarding preferences of experts who do not contribute to achieve consensus [5] or penalizing their importance weights, thus reducing their influence in the CRP [17], [26]. Here, a weight penalizing method is proposed so that the weights of noncooperating experts' preferences are reduced accordingly throughout the discussion process.

As mentioned in the consensus model scheme in Section IIIA, each expert $e_{i} \in E$ has an associated importance weight $w_{i} \in[0,1]$. At the beginning of the CRP, all experts have a maximum weight, $w_{i}=1, \forall i$, and such a weight could be updated whenever a behavior detection occurs.

Given a cluster $C_{h}^{t}$ which contains a con-coalition in round $t \geq 2$, the procedure shown in Algorithm 2 is applied to each expert preference relation $P_{i}^{t} \in C_{h}^{t}$ to update its corresponding weight $w_{i}$. The procedure to manage individual behaviors 


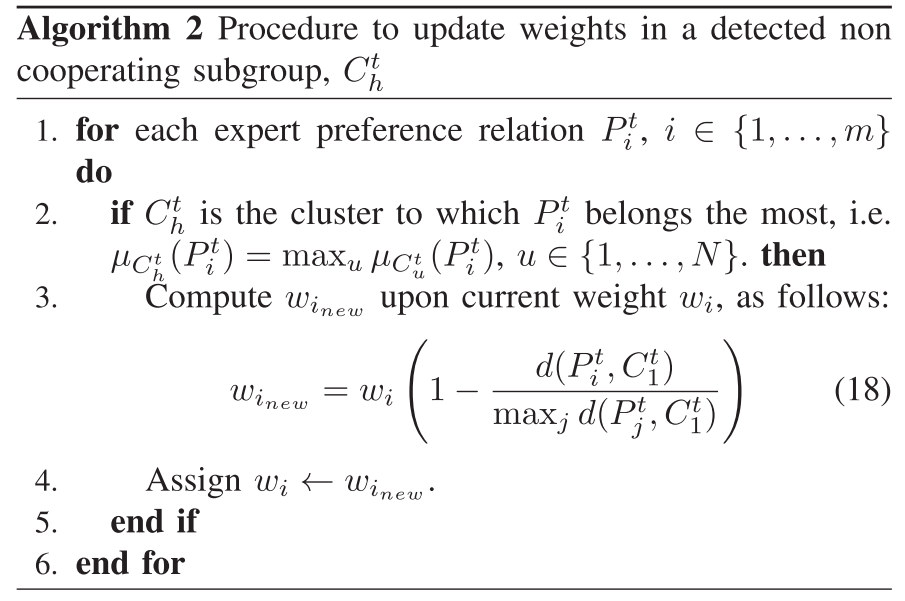

(outliers) consists in applying steps 3 and 4 of Algorithm 2 for a detected $P_{i}^{t}$.

Equation (18) is used to obtain the updated weight for $e_{i}$, i.e., $w_{i_{\text {new }}}$, based on its current weight $w_{i}$, the distance to the collective preference $C_{1}^{t}$, and the maximum distance between an expert's preference and $C_{1}^{t}$, i.e., $\max _{j} d\left(P_{j}^{t}, C_{1}^{t}\right)$. This expression ensures that $w_{i_{\text {new }}}$ is bounded to the $\left[0, w_{i}\right] \subseteq[0,1]$ interval and $w_{i_{\text {new }}} \leq w_{i}$. Notice here that if $P_{i}^{t}$ is the furthest preference relation from $P_{c}^{t}$, then $d\left(P_{i}^{t}, C_{1}^{t}\right)=\max _{j} d\left(P_{j}^{t}, C_{1}^{t}\right)$, and consequently, $w_{i_{\text {new }}}=0$; therefore, $e_{i}$ 's importance weight becomes null.

As previously shown in Section III-A, the reduction of experts' importance weights in round $t$ affects two steps in the following round $t+1$ of the CRP.

1) The computation of the consensus matrix $C M$ upon experts' similarities.

2) The computation of $P_{c}$ upon experts' preference relations.

Regarding the former step, since $C M$ is obtained by aggregating similarity values $s m_{i j}^{l k}$ for each pair of experts, it is necessary to combine $w_{i}$ and $w_{j}$ in order to obtain a weight $w_{i j}$ associated with such a pair. It is assumed that if at least one expert weight in the pair $\left(e_{i}, e_{j}\right)$ has been penalized, then the importance weight $w_{i j}$ assigned to their similarity degree $s m_{i j}^{l k}$ should be decreased. Therefore, it is proposed computing the weight of the pair $\left(e_{i}, e_{j}\right)$ as $w_{i j}=\min \left(w_{i}, w_{j}\right)$.

Finally, as it will be shown in the illustrative example in Section V, two different weight penalizing schemes can be defined.

1) Partial weight penalizing: Reduced weights are taken into account in the computation of $P_{c}$ only [see (8)], with the aim of making $P_{c}$ closer to the preferences of those experts who contribute to achieving a consensus.

2) Full weight penalizing: It is an extended case of the partial weight penalizing where, besides considering reduced weights to compute $P_{c}$, the agreement positions of those experts who contribute to achieving a consensus are also taken into account, in order to improve the convergence in the consensus degree, $\mathrm{cr}$. Therefore, reduced weights are also integrated in the computation of $C M$ [see (4)].

The effect of using either one of these penalizing schemes in the CRP will be shown in Section V. Fig. 6 shows graphically the overall process to manage noncooperating experts.

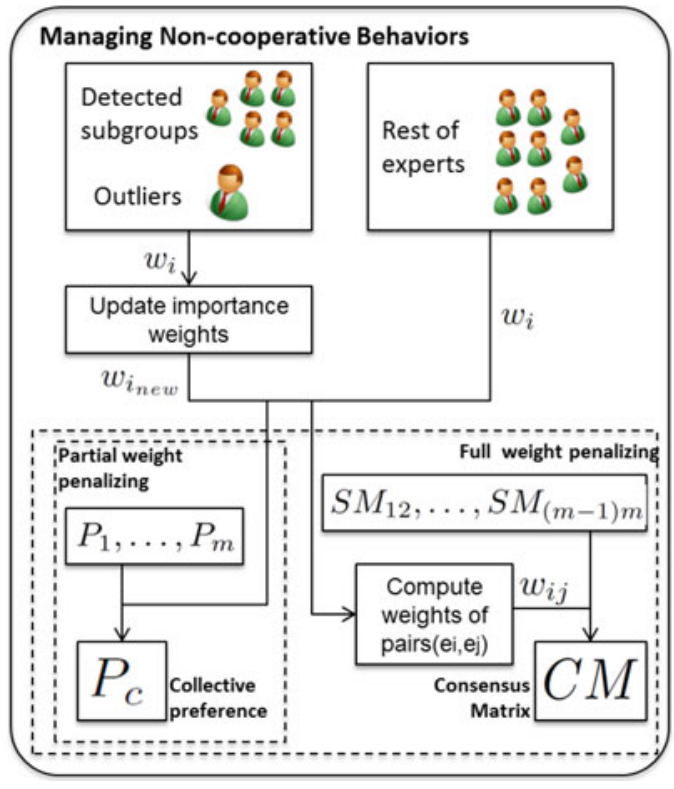

Fig. 6. Scheme of the method to manage noncooperating experts in CRPs.

\section{Monitoring Tool Based on Self-Organizing Maps}

Besides the proposed consensus model, and due to the necessity of having a visual insight on experts' preferences and their evolution across the CRP, in this section, we propose a monitoring tool based on SOMs. Such a tool can be considered a complement to the consensus model presented in the previous section, which not only provides a clearer vision of the CRP performance, but also lets us find experts and subgroups of experts who may present different patterns of behavior against consensus, due to the fact that their preferences are moved against the collective opinion.

Different applications and tools have been implemented to support the SOM-based visualization of high-dimensional data. One of them is SOM Toolbox, ${ }^{1}$ i.e., a powerful research-oriented plug-in for the widely known MATLAB ${ }^{2}$ software suite [20], which provides multiple ways of visualizing data, for instance, by means of their 2-D PCA projection. SOM Toolbox can be used to process and visualize experts' preference relations and cluster centers managed by the consensus model proposed in this paper. To do so, we propose the following procedure, as depicted in Fig. 7, which is applied at the end of each CRP round $t$ :

1) The collective preference $P_{c}$ and cluster centers $C_{h}$ in the current round are computed from experts' preferences $P_{i}$, as explained in Section III.

2) All preference relations, including $P_{c}$ and cluster centers, are gathered into a so-called preference-cluster dataset file, where each data object is a preference relation, which is represented as a vector of dimension $n \times n$. The first line of the dataset contains a number indicating the dimension of data. Data objects corresponding with cluster centers are given the label "C," whereas the collective preference

\footnotetext{
${ }^{1}$ http://www.cis.hut.fi/somtoolbox/

${ }^{2}$ http://www.mathworks.com
} 


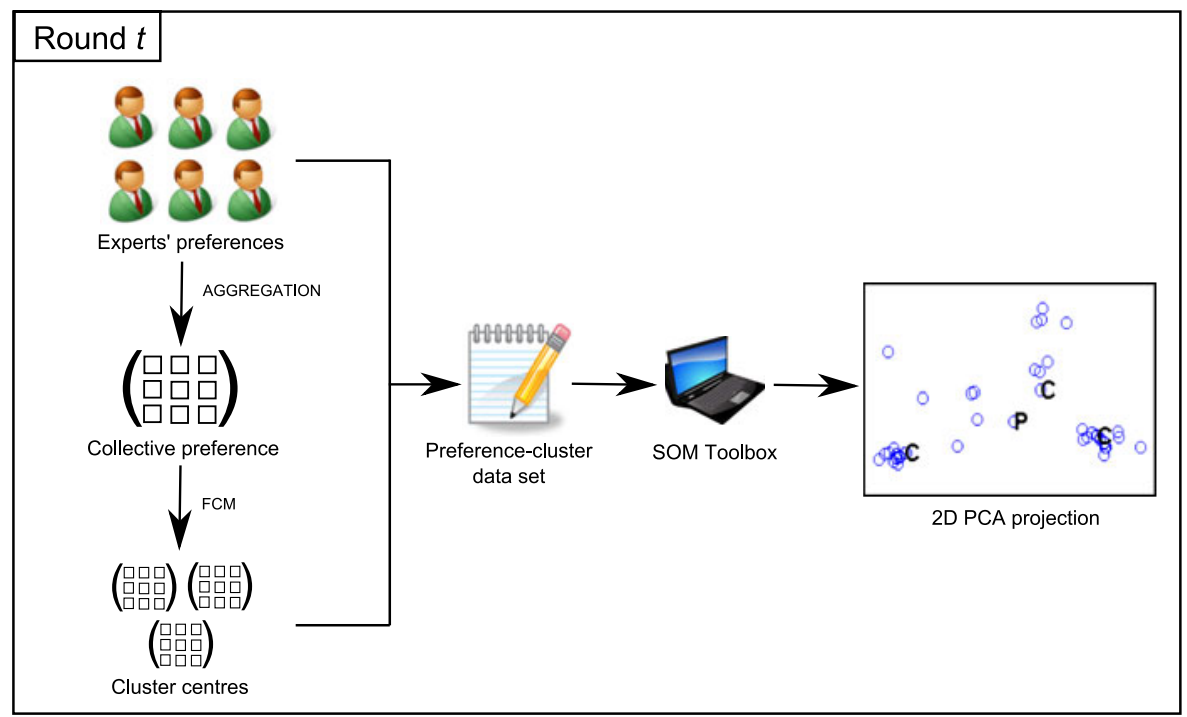

Fig. 7. Process to visualize experts' preferences and cluster centers in a CRP round $t$.

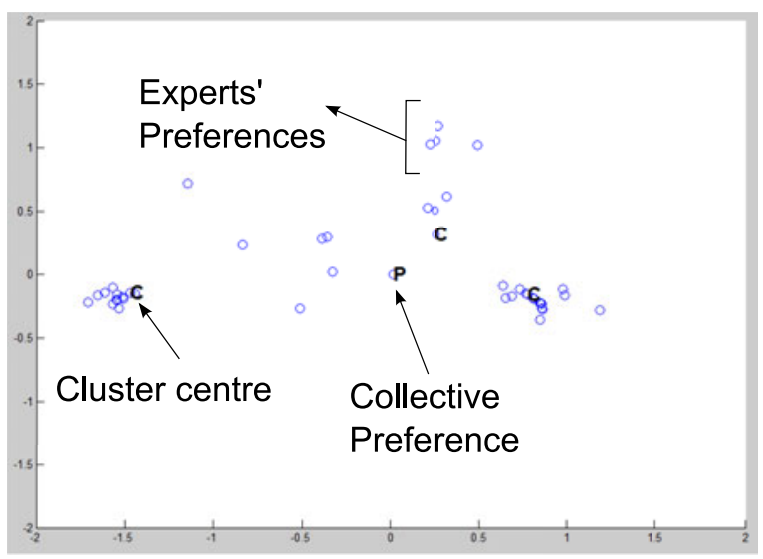

Fig. 8. Two-dimensional visualization of experts' preferences and clusters with SOM Toolbox.

is given the label "P" so that they can be easily localizable in the visual representation of preferences.

3) The preference-cluster dataset is processed by SOM Toolbox to generate a 2-D PCA projection of experts' preferences and cluster centers in the current CRP round.

Fig. 8 shows an example of 2-D visualization of experts' preferences and clusters, generated with SOM Toolbox.

\section{ILLUSTRATIVE EXAMPLE}

In this section, an implemented version of the presented consensus model is used to solve a real-life GDM problem where a high number of decision makers participate. The main goal of such a simulation is to show the effectiveness and usefulness of our proposal when dealing with large groups of experts, some of which might present noncooperative behaviors during the consensus process, thus hindering the achievement of an agreement.

The problem formulation is as follows: Let us suppose that an expert commission compound by 50 members belonging to different areas $E=\left\{e_{1}, \ldots, e_{50}\right\}$ must make an agreed decision regarding a recent discovery of fossil fuels in the province of Jaén, in Andalucía, Spain. The proposed alternatives $X=\left\{x_{1}, x_{2}, x_{3}, x_{4}\right\}$ are the following ones.

1) $x_{1}$ : Discard any exploitation actions, due to environmental factors.

2) $x_{2}$ : Authorize a national company to search for natural gas sources.

3) $x_{3}$ : Authorize a multinational company to search for oil.

4) $x_{4}$ : Do a previous research on the area led by regional government.

The commission must achieve a minimum level of agreement of $\mu=0.85$ before making a decision; the maximum number of rounds of discussion allowed is Maxround $=10$, and the acceptability threshold is set as $\varepsilon=0.02$. Some experts in the group may present individual behaviors, or they may form coalitions with a noncooperative behavior, as it will be shown in the example.

Common parameters for the clustering, detection, and management of behaviors are set as follows:

1) Fuzziness coefficient: $b=2$.

2) Threshold for stopping condition in FCM: $\epsilon=0.001$.

3) Distance measure: Minkowski distance with $p=1$.

4) Cluster similarity threshold: $\kappa=0.9$.

5) Minimum detectable distance among clusters: $\nu=0.01$.

6) Consensus threshold to activate outlier detection, $\gamma=$ 0.75 .

7) Membership threshold for outliers, $\delta=0.4$.

Two experimental studies have been conducted. In the first one, the effects of applying the different penalizing schemes to manage behaviors are shown, whereas the second one focuses on analyzing the effects in the CRP of using different values for the number of clusters, $N$, in the FCM algorithm.

Remark 3: No comparison with other techniques is shown in this paper because, as far as we know, this is the first time a methodology based on fuzzy clustering is implemented and applied to support CRPs, and most current proposals of consensus 
TABLE I

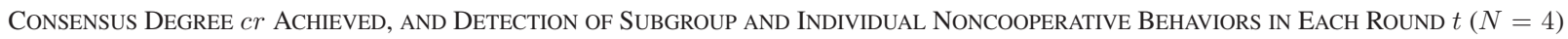

\begin{tabular}{|c|c|c|c|c|c|c|c|c|c|}
\hline$t$ & \multicolumn{3}{|c|}{ Without weight Penalizing } & \multicolumn{3}{|c|}{ Partial weight Penalizing } & \multicolumn{3}{|c|}{ Full weight Penalizing } \\
\hline & $\mathrm{cr}$ & $\begin{array}{c}\text { Subgroup } \\
\text { detected }\end{array}$ & $\begin{array}{c}\text { Outl. } \\
\text { detected }\end{array}$ & $\mathrm{cr}$ & $\begin{array}{c}\text { Subgroup } \\
\text { detected }\end{array}$ & $\begin{array}{c}\text { Outl. } \\
\text { detected }\end{array}$ & $\mathrm{cr}$ & $\begin{array}{c}\text { Subgroup } \\
\text { detected }\end{array}$ & $\begin{array}{c}\text { Outl. } \\
\text { detected }\end{array}$ \\
\hline 1 & 0.63060 & - & - & 0.63060 & - & - & 0.63060 & - & - \\
\hline 2 & 0.66655 & - & - & 0.66648 & - & - & 0.66614 & - & - \\
\hline 3 & 0.69543 & $\sqrt{ }$ & - & 0.69573 & $\sqrt{ }$ & - & 0.69473 & $\sqrt{ }$ & - \\
\hline 4 & 0.71914 & $\sqrt{ }$ & - & 0.71953 & $\sqrt{ }$ & - & 0.74663 & $\sqrt{ }$ & - \\
\hline 5 & 0.72266 & $\sqrt{ }$ & - & 0.72605 & $\sqrt{ }$ & - & 0.84651 & $\sqrt{ }$ & 3 \\
\hline 6 & 0.73317 & - & - & 0.75277 & $\sqrt{ }$ & 3 & 0.85743 & & \\
\hline 7 & 0.73525 & - & - & 0.78426 & $\sqrt{ }$ & 1 & & & \\
\hline 8 & 0.74312 & - & - & 0.80119 & $\sqrt{ }$ & 1 & & & \\
\hline 9 & 0.74468 & $\sqrt{ }$ & - & 0.80174 & $\sqrt{ }$ & 1 & & & \\
\hline 10 & 0.74427 & & & 0.80160 & & & & & \\
\hline
\end{tabular}

models are for small groups and they do not focus on large-scale GDM.

\section{A. Experiments With Different Penalizing Schemes}

First, the model is used to solve the GDM three times, applying the behavior detection scheme in all of them (with $N=n=4$ clusters; see Section III-B), and different variations in the behavior management scheme for each one (see Section III-C):

1) Without weight penalizing: No penalizing is conducted upon detection.

2) Partial weight penalizing: A penalization on experts' weights is conducted only when computing the collective preference, $P_{c}$.

3) Full weight penalizing: A penalization on experts' weights is conducted when computing $P_{c}$ and the consensus matrix, $C M$, from experts' similarity values.

Our hypothesis states that the application of behavior detection and management schemes on experts' preferences might improve the CRP performance by increasing the convergence of $c r$ toward the desired level of agreement, $\mu$ :

a) A partial weight penalizing may cause $P_{c}$ to become closer to those experts who behave in favor of consensus, thus increasing slightly the convergence of $\mathrm{cr}$ toward $\mu$.

b) A full weight penalizing may also take into account rather those experts who contribute to achieve an agreement in the computation of $C M$, which might imply a more substantial increase in the convergence of $\mathrm{cr}$.

Once conducted the CRP, results are shown and analyzed. Table I shows the evolution of the consensus degree $\mathrm{cr}$ in each round, as well as the detection of subgroup and individual (outlier) behaviors, for each resolution of the GDM problem. The convergence of $c r$ during the CRP is also graphically shown in Fig. 9. In the cases of applying null or partial penalizing, consensus is not achieved; therefore, it is necessary to apply a full penalizing to achieve it, by assigning low importance weights to noncooperating individuals and subgroups not only when computing $P_{c}$, but also when obtaining consensus degrees.

In order to provide a visual monitoring of the overall CRP performance, the SOM-based visualization tool SOM Toolbox

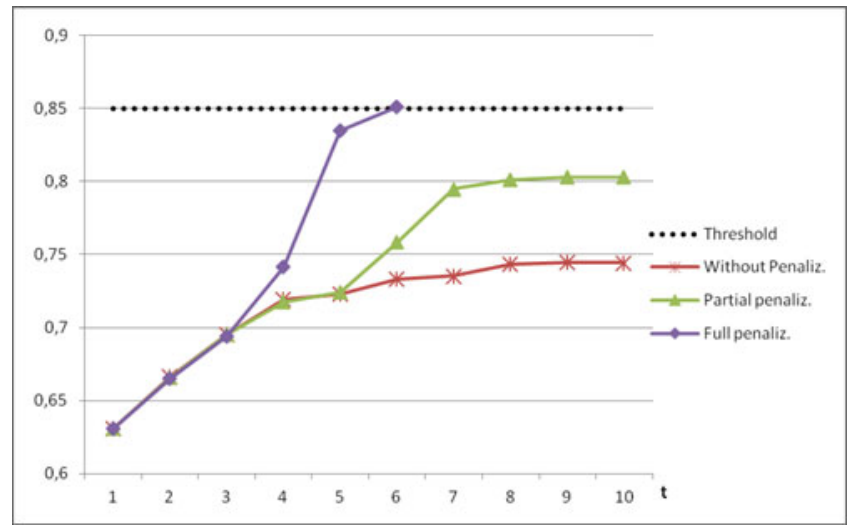

Fig. 9. Evolution of consensus degree $\mathrm{cr}$ in each round.

is used to show experts' preferences in a 2-D plot, as explained in Section IV. For the sake of space, we show the monitoring of the whole CRP for the case of applying full penalization. Fig. 10 shows the visual representation of experts' preferences, the collective preference $P_{c}$ (in the figure, denoted by "P"), and cluster centers $C_{h}$ (in the figure, denoted by "C") for each round of the CRP. As can be seen, a con-coalition of noncooperating experts is first detected at the end of the third round and, consequently, penalized from the fourth round onwards (solid rectangles represent penalized subgroups). When $\gamma$ is exceeded, outliers (i.e., individual noncooperative behaviors) are also detected and their weight is reduced (in the figure, they are surrounded by dashed rectangles). Additionally, from the fourth discussion round onwards, the position of $P_{c}$ in the SOM shifts from the center of the SOM, which means that the weights of experts' preferences, which are used in the computation of $P_{c}$, have been updated due to penalizing, favoring those experts who contribute positively to achieve a consensus.

Remark 4: The SOMs do not represent the absolute position of preference values, but rather the relative closeness of preferences among each other. Therefore, a $P_{c}$ in the center of several plots [see, e.g., Fig. 10(a)-(c)] does not indicate equal values of $P_{c}$ in them, but rather a collective preference obtained by using (9) (before penalizing weights).

Finally, Fig. 11 shows the visual representation of experts in the final round for each one of the three cases studied. It is 


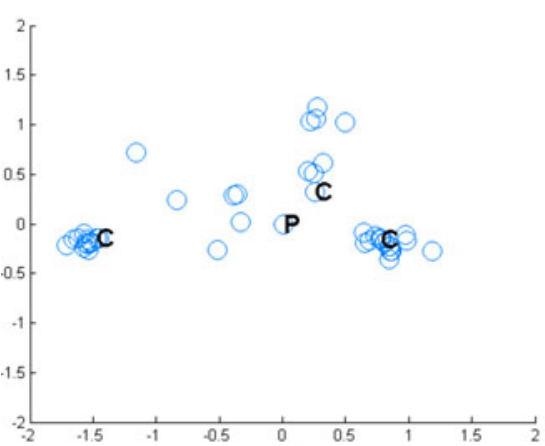

(a)

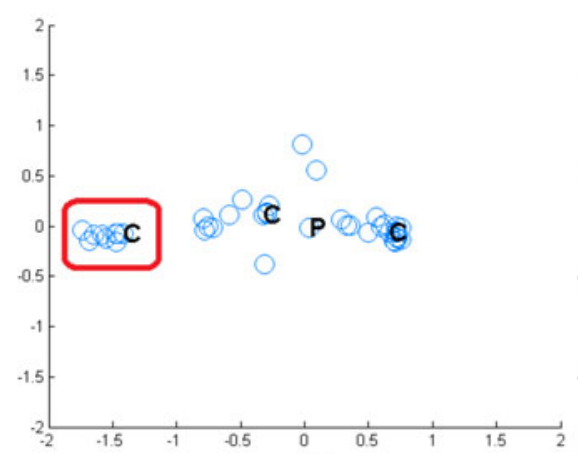

(d)

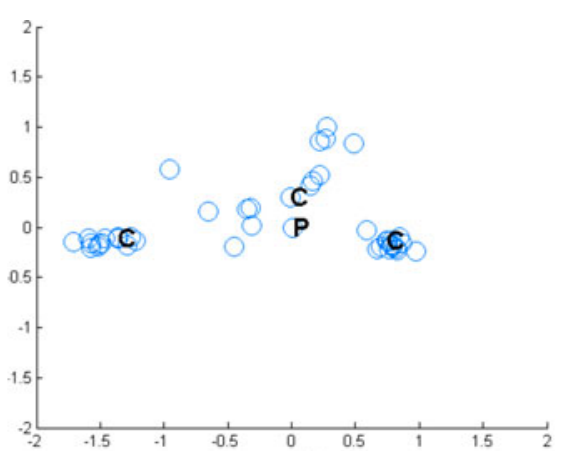

(b)

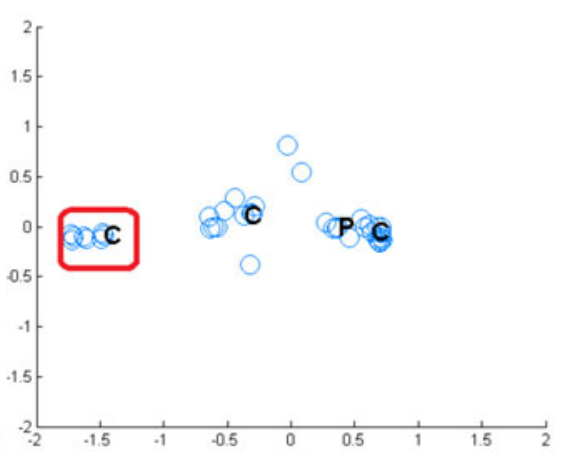

(e)

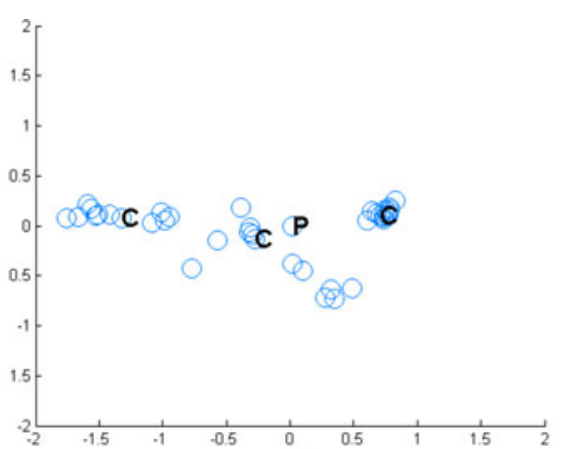

(c)

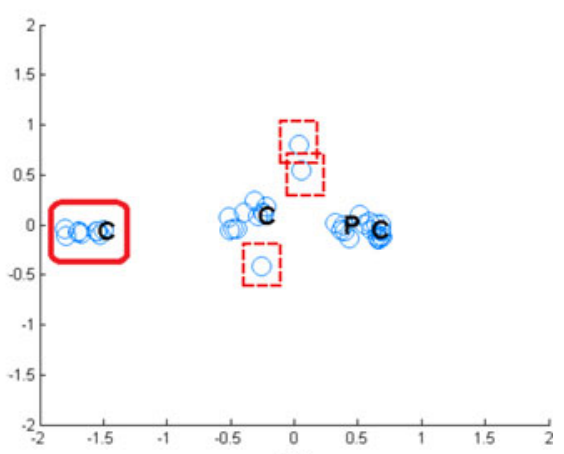

(f)

Fig. 10. Experts' preferences visualization during the CRP, with full weight penalizing. (a) Round 1 ( $c r=0.63060$ ). (b) Round 2 ( $c r=0.66614$ ). (c) Round 3 $(c r=0.69743)$. (d) Round 4 ( $c r=0.74663)$. (e) Round 5 ( $c r=0.84651)$. (f) Round 6 ( $c r=0.85743)$.

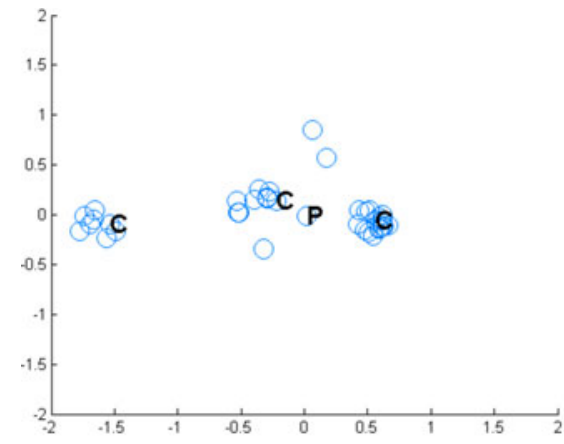

(a)

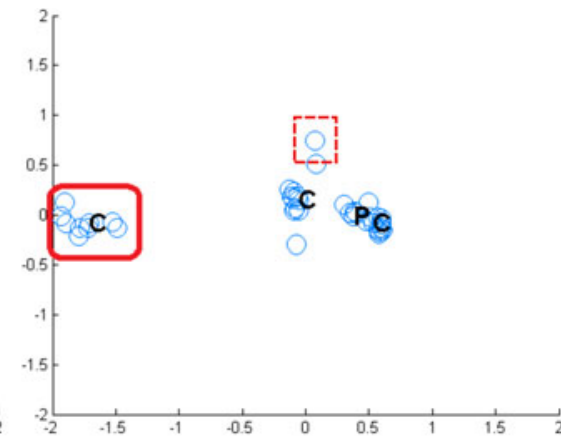

(b)

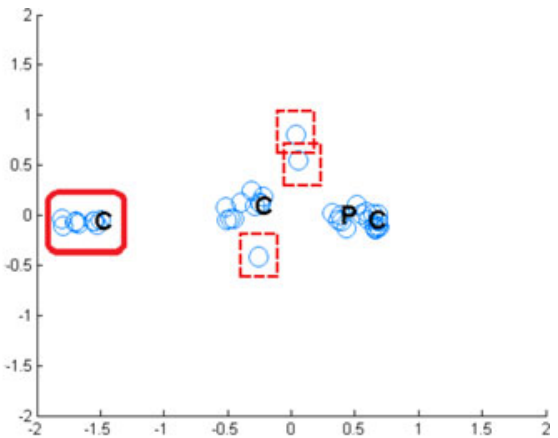

(c)

Fig. 11. Visualization of experts' preferences in the last round of the CRP. (a) No penalizing $(t=10, c r \simeq 0.744)$. (b) Partial penalizing $(t=10, c r \simeq 0.802$ ). (c) Full penalizing $(t=6, c r \simeq 0.857)$.

remarkable here how the application of any of the two proposed weight penalizing schemes affects the value of $P_{c}$, which is moved with respect to the case of no penalizing, becoming closer to the opinions of those experts who contribute to achieving an agreement and further from the opinions of noncooperating experts. This may affect the subsequent alternative selection process and the final decision made. A similar position of $P_{c}$ is obtained for both types of penalizing, since the main effect of applying a full penalizing with respect to a partial one is a higher convergence of $c r$.

These results allow us to confirm the hypothesis formulated, thus showing the importance and effectiveness of our approach to deal with large groups of decision makers, some of which might move their preferences against consensus and would pre- vent achieving the desired level of agreement if they are not detected and managed accordingly.

\section{B. Experiments With Different Number of Clusters}

Finally, some additional experiments are carried out by solving the consensus process with identical parameters, applying a full weight penalizing and using different values for the number of clusters considered in the FCM algorithm, i.e., $N$.

Table II shows the consensus degrees and detected behaviors for different values of $N$, and Fig. 12 illustrates the position of cluster centers obtained in the first CRP round. From experiments conducted, we can conclude the following. 
TABLE II

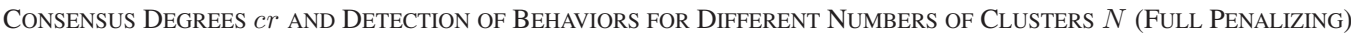

\begin{tabular}{|c|c|c|c|c|c|c|c|c|c|}
\hline$t$ & \multicolumn{3}{|c|}{$\mathrm{N}=3$} & \multicolumn{3}{|c|}{$\mathrm{N}=5$} & \multicolumn{3}{|c|}{$\mathrm{N}=8$} \\
\hline & $\mathrm{cr}$ & $\begin{array}{c}\text { Subgroup } \\
\text { detected }\end{array}$ & $\begin{array}{c}\text { Outl. } \\
\text { detected }\end{array}$ & $\mathrm{cr}$ & $\begin{array}{c}\text { Subgroup } \\
\text { detected }\end{array}$ & $\begin{array}{c}\text { Outl. } \\
\text { detected }\end{array}$ & $\mathrm{cr}$ & $\begin{array}{c}\text { Subgroup } \\
\text { detected }\end{array}$ & $\begin{array}{c}\text { Outl. } \\
\text { detected }\end{array}$ \\
\hline 1 & 0.63060 & - & - & 0.63060 & - & - & 0.63060 & - & - \\
\hline 2 & 0.66638 & - & - & 0.66853 & - & - & 0.66729 & $\sqrt{ }$ & - \\
\hline 3 & 0.70582 & - & - & 0.69869 & $\sqrt{ }$ & - & 0.73263 & $\sqrt{ }$ & - \\
\hline 4 & 0.72004 & $\sqrt{ }$ & - & 0.75141 & $\sqrt{ }$ & 6 & 0.75771 & $\sqrt{ }$ & 15 \\
\hline 5 & 0.83488 & $\sqrt{ }$ & 3 & 0.83502 & $\sqrt{ }$ & 5 & 0.78596 & $\sqrt{ }$ & 15 \\
\hline 6 & 0.85374 & & & 0.85065 & & & 0.81584 & $\sqrt{ }$ & 14 \\
\hline 7 & & & & & & & 0.82774 & $\sqrt{ }$ & 21 \\
\hline 8 & & & & & & & 0.82925 & $\sqrt{ }$ & 27 \\
\hline 9 & & & & & & & 0.82981 & $\sqrt{ }$ & 15 \\
\hline 10 & & & & & & & 0.83007 & $\sqrt{ }$ & 20 \\
\hline
\end{tabular}

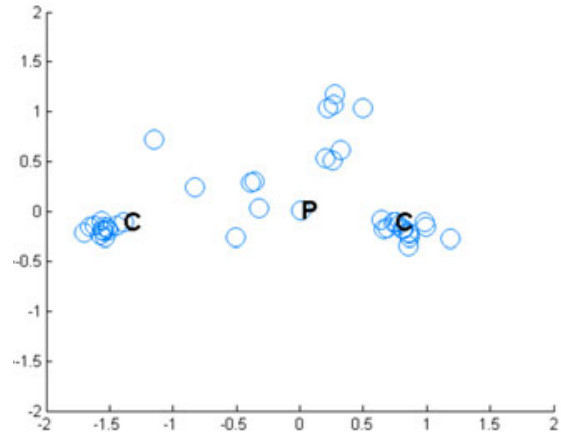

(a)

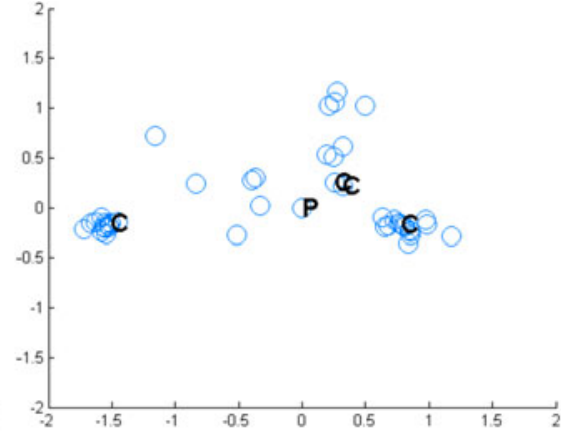

(b)

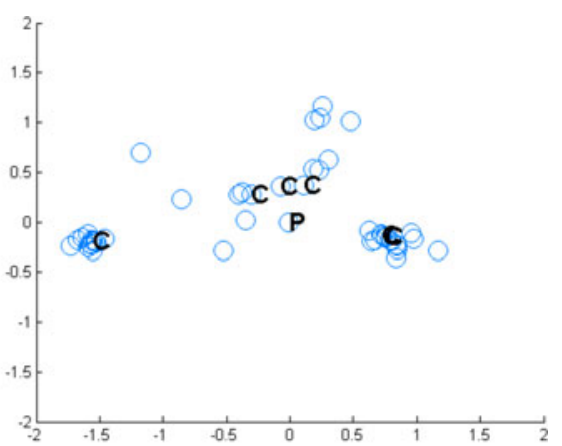

(c)

Fig. 12. Visualization of cluster centers in the first round of the CRP. (a) $N=3(t=1)$. (b) $N=5(t=1)$. (c) $N=8(t=1)$.

1) $N=2$ leads to undesired results, as $C_{2}$ always tends to approximate to $P_{c}$, which does not vary during the FCM algorithm; therefore, its use has been discarded.

2) If $N>n$ (with $n$ being the number of alternatives, in our case, $n=4$ ), then some cluster centers are close to each other and they tend to overlap as $N$ increases (see Fig. 12(c), where $N=8$ and where some cluster centers overlap). Moreover, if a too high value of $N$ is chosen, an excessive number of subgroup and individual misbehaviors are detected, which affects nearly all experts' weights during penalizing, and consequently, the convergence toward consensus is not improved with respect to applying no penalizing.

3) Values of $N$ which are close to the number of alternatives $n$ provide good results in the behavior detection and an adequate convergence toward consensus.

It is concluded that an appropriate value for $N$ is $n=4$ (as it was considered in Section V-A), which makes sense if we assume that different experts' in the group might have a predilection for each one of the distinct alternatives $x_{l} \in X$; hence, it is usual that at most $n$ different subgroups with a clear preference over an specific alternative might appear during the CRP.

\section{CONCLUDING REMARKS}

Current challenges for the improvement of CRPs in GDM include the necessity of developing new consensus models capable of managing large scales of decision makers effectively, thus overcoming the difficulties derived from the high cost, com- plexity, the constant human supervision, or even the possibility of dealing with subgroups of decision makers who present noncooperative behaviors during the discussion process. Real-life decision making problems involving a large number of decision makers are becoming increasingly common, as what occurs, for instance, with new trends such as e-democracy processes and social networks. In this paper, a consensus model capable of dealing with large groups of decision makers has been presented. Such a model utilizes an approach based on fuzzy clustering to detect and manage individuals or subgroups of decision makers who do not cooperate during the discussion process. Additionally, the model is complemented with a monitoring tool to visualize decision makers' preferences and their evolution during the CRP.

Despite the paper proposal has been presented under a methodological viewpoint, future works are mainly focused on developing a distributed software, which will be used to conduct a real large-scale experiment and prove the validity of the proposed model in a real-life problem. The proposed model is valid as such for its application in any business and organizational contexts, and it can also be easily extended by adapting it to more specific contexts, as mentioned previously. Other future works are focused on the use of the proposed methodology in linguistic decision making [46], [47].

\section{REFERENCES}

[1] J. Kacprzyk, "Group decision making with a fuzzy linguistic majority," Fuzzy Sets Syst., vol. 18, no. 2, pp. 105-118, 1986. 
[2] C. Butler and A. Rothstein, On Conflict and Consensus: A Handbook on Formal Consensus Decision Making. Takoma Park, MD, USA: Food Not Bombs, 2006.

[3] A. Tocqueville, Democracy in America (2nd. Edition). London, U.K.: Saunders and Otley, 1840.

[4] F. Herrera, E. Herrera-Viedma, and J. Verdegay, "A sequential selection process in group decision making with linguistic assessments," Inf. Sci., vol. 85 , no. 1995 , pp. 223-239, 1995.

[5] S. Saint and J. R. Lawson, Rules for Reaching Consensus. A Modern Approach to Decision Making. San Francisco, CA, USA: Jossey-Bass, 1994.

[6] L. Martínez and J. Montero, "Challenges for improving consensus reaching process in collective decisions," N. Mathemat. Natural Comput., vol. 3, no. 2, pp. 203-217, 2007.

[7] E. Herrera-Viedma, F. Herrera, and F. Chiclana, "A consensus model for multiperson decision making with different preference structures," IEEE Trans. Syst. Man Cybern. A, Syst. Humans, vol. 32, no. 3, pp. 394-402, Dec. 2002.

[8] E. Herrera-Viedma, L. Martínez, F. Mata, and F. Chiclana, "A consensus support system model for group decision making problems with multigranular linguistic preference relations," IEEE Trans. Fuzzy Syst., vol. 13, no. 5, pp. 644-658, Oct. 2005.

[9] J. Kacprzyk, M. Fedrizzi, and H. Nurmi, "Group decision making and consensus under fuzzy preferences and fuzzy majority," Fuzzy Sets Syst., vol. 49, no. 1, pp. 21-31, 1992.

[10] F. Mata, L. Martínez, and E. Herrera-Viedma, "An adaptive consensus support model for group decision-making problems in a multigranular fuzzy linguistic context," IEEE Trans. Fuzzy Syst., vol. 17, no. 2, pp. 279290, Jan. 2009

[11] R. Parreiras, P. Ekel, J. Martini, and R. Palhares, "A flexible consensus scheme for multicriteria group decision making under linguistic assessments," Inf. Sci., vol. 180, no. 7, pp. 1075-1089, 2010

[12] R. Efremov, D. Rios-Insua, and A. Lotov, "A framework for participatory decision support using pareto frontier visualization, goal identification and arbitration," Eur. J. Oper. Res., vol. 199, no. 2, pp. 459-467, 2009

[13] J. Kim, "A model and case for supporting participatory public decision making in e-democracy," Group Decis. Negot., vol. 17, no. 3, pp. 179-192, 2008.

[14] M. Squillante, "Decision making in social networks," Int. J. Intell. Syst., vol. 25, no. 3, 225 pp. (Spec. Iss.), 2010.

[15] C. Sueur, J. Deneubourg, and O. Petit, "From social network (centralized vs. decentralized) to collective decision-making (unshared vs. shared consensus)," PLoS one, vol. 7, no. 2, pp. 1-10, 2012.

[16] R. R. Yager, "Intelligent social network analysis using granular computing," Int. J. Intell. Syst., vol. 23, pp. 1197-1220, 2008.

[17] R. R. Yager, "Penalizing strategic preference manipulation in multi-agent decision making," IEEE Trans. Fuzzy Syst., vol. 9, no. 3, pp. 393-403, Jun. 2001

[18] T. Kohonen, Self-Organizing Maps. Heidelberg, Germany: Springer, 1995

[19] J. Vesanto, "SOM-based data visualization methods," Intell. Data Anal., vol. 3, no. 2, pp. 111-126, 1999.

[20] J. Vesanto, J. Himberg, E. Alhoniemi, and J. Parhankangas, "Selforganizing map in Matlab: The SOM toolbox," in Proc. Matlab DSP Conf., 2000, pp. 35-40.

[21] S. Orlovsky, "Decision-making with a fuzzy preference relation," Fuzzy Sets Syst., vol. 1, no. 3, pp. 155-167, 1978.

[22] H. Bustince, M. Pagola, R. Mesiar, E. Hullermeier, and F. Herrera, "Grouping, overlap and generalized bientropic functions for fuzzy modeling of pairwise comparisons," IEEE Trans. Fuzzy Syst., vol. 20, no. 3, pp. 405415, Jun. 2012.

[23] N. Bryson, "Group decision-making and the analytic hierarchy process. exploring the consensus-relevant information content," Comput. Oper. Res., vol. 23, no. 1, pp. 27-35, 1996.

[24] T. Tanino, "Fuzzy preference relations in group decision making," in Non-Conventional Preference Relations in Decision Making, J. Kacprzyk and M. Roubens, Eds. Berlin, Germany: Springer-Verlag, 1988, pp. 54 71.

[25] M. Roubens, "Fuzzy sets and decision analysis," Fuzzy Sets Syst., vol. 90, no. 2, pp. 199-206, 1997.

[26] E. Herrera-Viedma, J. García-Lapresta, J. Kacprzyk, M. Fedrizzi, H. Nurmi, and S. Zadrozny, Eds., Consensual Processes. Studies in Fuzziness and Soft Computing. vol. 267. New York, NY, USA: Springer, 2011.
[27] J. Kacprzyk and M. Fedrizzi, "A soft measure of consensus in the setting of partial (fuzzy) preferences," Eur. J. Oper. Res., vol. 34, no. 1, pp. 316-325, 1988.

[28] F. Herrera, E. Herrera-Viedma, and J. Verdegay, "A model of consensus in group decision making under linguistic assessments," Fuzzy Sets Syst. vol. 78, no. 1, pp. 73-87, 1996.

[29] J. Kacprzyk and S. Zadrozny, "Soft computing and web intelligence for supporting consensus reaching," Soft Comput., vol. 14, no. 8, pp. 833-846, 2010.

[30] M. Fedrizzi, M. Fedrizzi, and R. Marques, "Soft consensus and network dynamics in group decision making," Int. J. Intell. Syst., vol. 14, no. 1, pp. 63-77, 1999.

[31] G. Beliakov, A. Pradera, and T. Calvo, Aggregation Functions: A Guide for Practitioners. New York, NY, USA: Springer, 2007.

[32] S. Alonso, E. Herrera-Viedma, F. Chiclana, and F. Herrera, "A web based consensus support system for group decision making problems and incomplete preferences," Inf. Sci., vol. 180, no. 23, pp. 4477-4495, 2010.

[33] S. Alonso, I. Pérez, F. Cabrerizo, and E. Herrera-Viedma, "A linguistic consensus model for web 2.0 communities," Appl. Soft Comput., vol. 13, no. 1 , pp. 149-157, 2013.

[34] Z. Xu and X. Cai, "Group consensus algorithms based on preference relations," Inf. Sci., vol. 181, no. 1, pp. 150-162, 2011.

[35] I. Palomares, R. Rodríguez, and L. Martínez, "An attitude-driven web consensus support system for heterogeneous group decision making," Expert Syst. Appl., vol. 40, no. 1, pp. 139-149, 2013.

[36] W. Pedrycz, "Clustering and Fuzzy Clustering," in Knowledge-Based Clustering. New York, NY, USA: Wiley, 2005, pp. 1-27.

[37] V. Cherkassky and F. Mulier, Learning From Data: Concepts, Theory and Methods. New York, NY, USA: Wiley, 2007.

[38] J. MacQueen, "Some methods for classification and analysis of multivariate observations," in Proc. 5th Berkeley Symp. Mathemat. Statist. Probabil., Berkeley, CA, USA, 1967, pp. 281-197.

[39] G. Klir and B. Yuan, Fuzzy Sets and Fuzzy Logic: Theory and Applications Englewood Cliffs, NJ, USA: Prentice-Hall, 1995.

[40] A. Baraldi and P. Blonda, "A survey of fuzzy clustering algorithms for pattern recognition-Part I," IEEE Trans. Syst. Man Cybern. B, Cybern., vol. 29, no. 6, pp. 778-785, Dec. 1999.

[41] J. Bezdek, Pattern Recognition With Fuzzy Objective Function Algorithms. New York, NY, USA: Wiley, 2007.

[42] M. Al-Daoud and S. Roberts, "New methods for the initialization of clusters," Pattern Recognit. Lett., vol. 17, no. 5, pp. 451-455, 1996.

[43] I. Katsavounidis, C. C. J. Kuo, and Z. Zhang, "A new initialization technique for generalized Lloyd iteration," IEEE Signal Process. Lett., vol. 1, no. 10, pp. 144-146, Oct. 1994.

[44] J. Vesanto and E. Alhoniemi, "Clustering of the self-organizing map," IEEE Trans. Neural Netw., vol. 11, no. 3, pp. 586-600, May 2000.

[45] M. Van-Hulle, "Self-organizing maps," in Self-Organizing Maps: Theory, Design, and Application. Tokyo, Japan: Kaibundo, 2011.

[46] R. Rodríguez, L. Martínez, and F. Herrera, "Hesitant fuzzy linguistic term sets for decision making," IEEE Trans. Fuzzy Syst., vol. 20, no. 1, pp. 109 119, Feb. 2012.

[47] F. Petry and R. Yager, "A linguistic approach to influencing decision behavior," IEEE Trans. Fuzzy Syst., vol. 20, no. 2, pp. 248-261, Apr 2012.

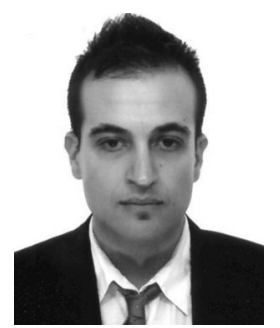

Iván Palomares received the M.Sc. degree in computer science from the University of Jaén, Jaén, Spain, in 2009 and the post-graduate master degree in soft computing and intelligent systems from the University of Granada, Granada, Spain, in 2011. He is currently working toward the Ph.D. degree with the Department of Computer Science, University of Jaén.

His research interests include decision making, consensus reaching, fuzzy logic, recommender systems, and multiagent systems.

Mr. Palomares received the Extraordinary Academic Degree Award and a National Award to Academic Performance, granted by the Faculty of Engineering, University of Jaén, and the Spanish Ministry of Education, respectively, for the M.Sc. degree in computer science. 


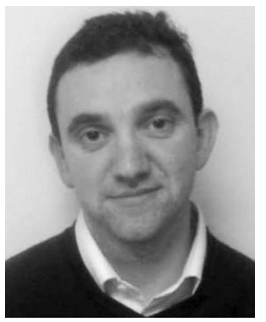

Luis Martínez (M'10) received the M.Sc. and Ph.D. degrees in computer science from the University of Granada, Granada, Spain, in 1993 and 1999, respectively.

He is currently a Full Professor with the Department of Computer Science and the Director of the Advanced Research Center in IT, University of Jaén, Jaén, Spain. He has been a supervisor of seven Ph.D. students and published more than 60 papers in international journals. His current research interests include computing with words and decision making, fuzzy logic-based systems, multiagent systems, computer-aided learning, sensory evaluation, recommender systems, and e-commerce.

Dr. Martínez is the Editor-in-Chief of the International Journal of Computational Intelligence Systems, an Associated Editor of the International Journal of Fuzzy Systems, and a member of the journal Editorial Board of the Journal of Universal Computer Sciences. He received the IEEE TRANSACTIONS ON FUZZY SYSTEMS Outstanding 2008 Paper Award (bestowed in 2011).

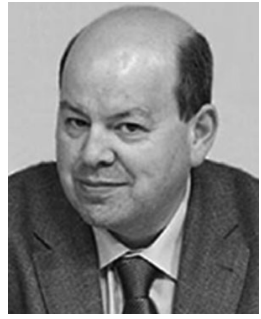

Francisco Herrera (M'10) received the M.Sc. and Ph.D. degrees in mathematics from the University of Granada, Granada, Spain, in 1988 and 1991, respectively.

He is currently a Professor with the Department of Computer Science and Artificial Intelligence, University of Granada. He has been the supervisor of 28 Ph.D. students. He has published more than 240 papers in international journals. He is coauthor of the book Genetic Fuzzy Systems: Evolutionary Tuning and Learning of Fuzzy Knowledge Bases (Singapore:

World Scientific, 2001). His current research interests include computing with words and decision making, data mining, bibliometrics, big data, data preparation, instance selection, fuzzy rule-based systems, genetic fuzzy systems, knowledge extraction based on evolutionary algorithms, memetic algorithms, and genetic algorithms.

Dr. Herrera is the Editor-in-Chief of the international journal Progress in Artificial Intelligence (Springer). He acts as an Area Editor of the International Journal of Computational Intelligence Systems and an Associated Editor of the following journals: the IEEE TRANSACTIONS ON FUZZY SYSTEMS, Information Sciences, Knowledge and Information Systems, Advances in Fuzzy Systems, and the International Journal of Applied Metaheuristics Computing. He serves as a member of several journal editorial boards, including Fuzzy Sets and Systems, Applied Intelligence, Information Fusion, Evolutionary Intelligence, the International Journal of Hybrid Intelligent Systems, Memetic Computation, and Swarm and Evolutionary Computation. He has received the following honors and awards: ECCAI Fellow 2009, IFSA 2013 fellow, 2010 Spanish National Award on Computer Science ARITMEL to the Spanish Engineer on Computer Science, International Cajastur Mamdani Prize for Soft Computing (Fourth Edition, 2010), the IEEE TRANSACTIONS ON FuZZY SySTEMS Outstanding 2008 Paper Award (bestowed in 2011), and the 2011 Lotfi A. Zadeh Prize Best Paper Award from the International Fuzzy Systems Association. 